\title{
COMPRENSIÓN DE CONCEPTOS FÍSICOS MEDIANTE EXHIBICIONES INTERACTIVAS: UN ESTUDIO COMPARATIVO
}

\author{
SEBASTIÁ, J.M. \\ Departamento de Física. Universidad Simón Bolívar. Apdo 89.000. Caracas 1080. Venezuela.
}

\begin{abstract}
SUMMARY
In this work we tried to find relationships between the variables of gender, age, aptitude, previous attitude and learning of a sample of 126 students between the ages of 14 and 16 in a visit to the Museo de Ios Niños, Caracas, Venezuela. We also carried out a comparative study between learning at the Museum and learning in a traditional classroom lecture, for two different groups. An Analysis of Variance showed a significant relation between previous attitude and age (inverse) and student learning. No significant differences were found between genders in the post test. Results show that the visit to the Museum was found to be more effective on learning than the classroom lecture. However, no differences were found between groups subjected to opposite order of treatment (Museum-Classroom lecture) through a counter-balance design approach.
\end{abstract}

\section{INTRODUCCIÓN}

Los museos de ciencias llevan a cabo una importante función educativa, la cual complementa la enseñanza formal que los estudiantes adquieren en la escuela. La enser̃anza informal que proporcionan estos museos se realiza tanto en las visitas individuales o en grupos familiares como en las visitas guiadas de grupos de escolares de una determinada institución.

Esta enseñanza informal, fuera de las instituciones escolares, está adquiriendo una relevancia cada vez mayor, ya que, en el cambiante mundo tecnológico actual, la formación científica no puede reducirse a los años escolares, sino que descansa cada vez más en una actualización de conocimientos a lo largo de toda la vida (Lucas, 1983; Feher y Rice, 1985; Lucas y McManus, 1986; Wellington, 1990; Stevenson, 1991; Culotta, 1992;
Tuckey; 1992). Complementariamente, los museos de ciencias han comprendido este desafío y están adaptándose a esta nueva función social, elaborando atractivos entornos de exhibiciones que estimulan el aprendizaje.

El efecto que tienen las visitas ha sido estudiado por distintos autores (Flexer y Borum, 1984; Prats, 1985; Duesing, 1986; Finson y Enochs, 1982; Eratuuli y Sneider 1990; Price y Hein, 1991; MacDonald y Silverstone, 1992), habiéndose encontrado evidencias del impacto que tienen en motivar y alentar cambios de actitudes cientificas en los estudiantes. Sin embargo, las investigaciones acerca del efecto en la comprension de Ia ciencia que tienen las visitas a los museos no ha conducido a resultados tan concluyentes, e incluso ha 
Los pretest y postest fueron aplicados en las clases habituales de la institución educativa una semana antes y una semana después de los tratamientos. Los intrumentos permitieron recoger Ios siguientes datos: el pretest (T1) permitió determinar las características de cada uno de los estudiantes en referencia a su edad, sexo, y actitud y aptitud previas; el postest (T2) determinó los resultados de cada estudiante después de cada uno de los tratamientos (post museo, PMU) y post clase (PCLS), la variación en la puntuación (GAN), y la opinión del estudiante sobre la utilidad de cada uno de ellos: satisfacción post museo (SMU) y satisfacción post clase (SCLS).

Los resultados de actitudes (variables: actitud, SMU, SCLS) fueron evaluados en base a una escala de 3 puntos, correspondiendo el puntaje de 3 a «muy satisfecho», y el de 1 a «poco satisfecho». La aptitud está medida en una escala de 6 puntos, y los postests de comprensión (variables: PMU, PCLS, GAN) están medidos en una escala de 10 puntos. La edad está expresada en años.

\section{Análisis de los datos}

Para el análisis de los datos recogidos en la investigación fucron empleados los siguientes tipos de técnicas estadísticas:

Un estudio comparativo, mediante el t-test, permitió comparar los resultados entre los dos grupos ( $\mathrm{G} 1$ y G2) en referencia a los puntajes post museo y post clase y a la satisfación del estudiante en cada tratamiento.

Un estudio de correlacion permitio detectar el sentido de Ia relación entre las variables independientes (sexo, edad, aptitud y actitud previa) y dependientes (puntajes de los tests post museo y post clase, satisfacción post museo y post clase), así como el grado de correlación entre las diferentes variables.

El análisis de la relación entre cada una de las variables independientes y los resultados de los tratamientos fueron llevados a cabo mediante un análisis de varianza simple (one way ANOVA). Un estudio post hoc, mediante el test de Scheffé fue aplicado a los casos en Ios que se detectaron diferencias signficativas. Un ańlisis de varianza múltiple (MANOVA) fue llevado a cabo para estudiar la interacción conjunta de varias variables independientes
Para todos los análisis en los cuales las comparaciones pudieron ser evaluadas en cuanto a su significación estadística, se eligió un nivel alfa del 0,05 .

\section{RESULTADOS}

\section{Diferencias generales entre los grupos antes y después de los tratamientos}

Los resuitados de puntajes obtenidos por los estudiantes de los dos grupos en las diferentes pruebas son los que se indican en la tabla $\mathbf{I}$.

Los resultados obtenidos indican que el grupo 1, que visit 6 primero el museo y luego recibio la clase, comenzó puntuando más alto que el grupo 2 ( $\mathrm{PMU}=7,22$ ), pero tuvo una menor ganancia entre el primer tratamiento y el segundo $(\mathrm{GAN}=0,55)$ para terminar después de los dos tratamientos con una puntuación: $\mathrm{PCLS}=7,77$. Por el contrario, el grupo 2, que primero recibió la clase y luego visitó el museo, comenzó puntuando más bajo después de Ia clase (PCLS=5,54), pero tuvo un aumento de puntuación mayor con el segundo tratamiento (GAN = 2,12 ), terminando los dos tratamientos con una puntuación prácticamente idéntica a la del grupo l (PMU = $7,66)$.

Un estudio de comparación, mediante un test del tipot, con un intervalo de confianza del $95 \%$, no detectó diferencias significativas respecto a las condiciones iniciales de ambos grupos; por lo tanto, los grupos fueron considerados estadísticamente equivalentes.

El estudio de comparación de resultados, mediante la $t$ de Student, no detectó diferencias significativas entre los test post museo (PMU) de los dos grupos $(t=-1,42$, $\mathrm{p}=0,159)$, pero sí las detectó entre los tests post clase, (PCLS, $\mathrm{t}=6,20, \mathrm{p}<0,001)$, en el sentido de que el tratamiento de la clase posterior a la visita al museo conduce a resultados más positivos que la clase previa a la visita al museo. Al parecer, influyó más la visita al museo para potenciar la clase que el procedimiento inverso.

Con referencia al grado de satisfaccion manifestada por los estudiantes después de cada tratamiento, el grupo l manifestó una similar actitud posterior a la visita al museo, $\mathrm{SMU}=2,22$ que el grupo $2, \mathrm{SMU}=2,12(\mathrm{t}=0,53$, $p=0,595$ ). Sin embargo, en referencia a la satisfación

\begin{tabular}{|lcccccccc}
\hline & $\begin{array}{c}\text { ACríuD } \\
\text { (base 3p) }\end{array}$ & $\begin{array}{c}\text { APTITUD } \\
\text { (base 6p) }\end{array}$ & $\begin{array}{c}\text { EDAD } \\
\text { (en años) }\end{array}$ & $\begin{array}{c}\text { PMU } \\
\text { (base 10p) }\end{array}$ & $\begin{array}{c}\text { PCLS } \\
\text { (base 10p) }\end{array}$ & $\begin{array}{c}\text { SMU } \\
\text { (basc 3p) }\end{array}$ & $\begin{array}{c}\text { SCLS } \\
\text { (base 3 p) }\end{array}$ & $\begin{array}{c}\text { GAN } \\
\text { (base 10p) }\end{array}$ \\
\hline Grupo 1 & 2,2985 & 4,3731 & 14,98 & 7,2221 & 7,7761 & 2,2273 & 1,8154 & 0,55 \\
Grupo 2 & 2,1695 & 4,3221 & 14,96 & 7,6652 & 5,5424 & 2,1724 & 1,5263 & 2,12 \\
\hline
\end{tabular}


Figura 1

Representación grafica de los resultados obtenidos en los dos tratamientos.

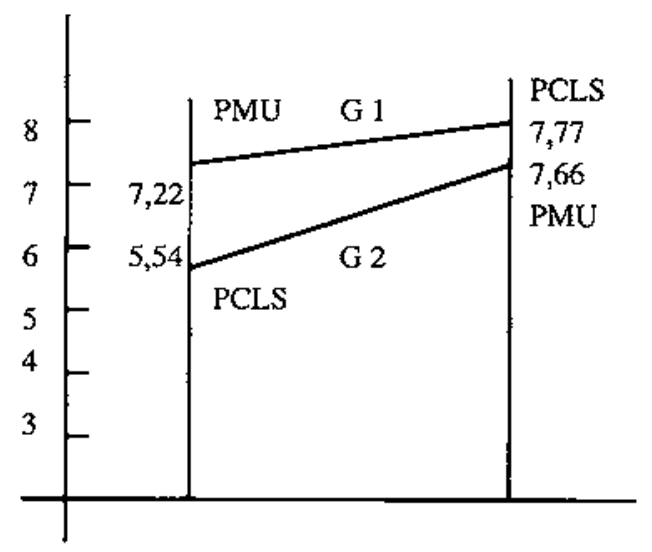

Fil grupo I siguió el procedimiento museo-clase. El grupo 2 siguió el procedimiento clase-museo.

con la clase, el grupo I, que recibió la clase después del museo, se manifestó más satisfecho con la clase $(\mathrm{SCLS}=1,81)$ que el grupo $2(\mathrm{SCLS}=1,53[t=0.309$, $\mathrm{p}<0.001]$ ), que recibió la clase antes de su visita al museo.

\section{Relaciones entre la edad y el sexo de los estudiantes con las características de aptitud y actitud de Ios estudiantes y con los resultados de los tratamientos}

Las edades de los sujetos pertenecientes a la muestra estuvieron distribuidas de la siguiente forma: 14 años $(25 \%), 15$ años $(52 \%)$ y 16 años $(23 \%)$. Las edades promedio de los grupos eran: grupo 1, edad promedio $=14,98$ años; grupo 2 , edad promedio $=14,97$ años.

Un análisis de varianza simple fue aplicado, con tos tres niveles de edades $(14,15$ y 16 años), para las características de entrada: aptitud y actitud previa de los dos grupos de la muestra, resultando diferencias significativas con la edad, tanto en la aptitud $(F[2,123]=$ $5,4701, \mathrm{p}=0,0053)$, como en la actitud ( $\mathrm{F}[2,123]=$ $6,4257, \mathrm{p}=0,0022)$.

El análisis post hoc, con un test de Scheffé, indicó que las diferencias son superiores al nivel alfa de 0,05 entre los niveles de edades 14 y 15 años y el nivel de 16 años en la aptitud; y entre el nivel de edad de 14 años y el nivel de 16 años, en la actitud. Estas diferencias de aptitud y actitud con la edad se reflejan en un coeficiente de correlación de Pearson negativo para ambas relaciones, edad-aptitud $(\mathrm{r}=-0,2607)$ y edad-actitud $(\mathrm{r}=-0,3068)$. Es decir, estas diferencias indican que, cuanto mayor es la edad del estudiante, más bajos sos sus puntajes de aptitud y actitud previa a los tratamientos.
En cuanto a la relaciones entre la edad y los resultados de Ios post tests de los tratamientos, se observó una influencia apreciabie, aunque no significativa al nivel alfa prefijado, únicamente para el test T2 post museo, $\mathrm{F}(2,123)=5,2961, p=0,062$. Esta diferencia de los resultados deI test $T 2$ post museo con la edad corresponde a un coeficiente de correlación negativo entre ambas variables $(r=-0,2494)$. Los estudiantes de mayor edad obtuvieron menores puntajes en el test post museo.

La variable sexo no mostró en ningún grupo una relación significativa con los conocimientos de circuitos eléctricos medidos con el test post clase, PCLS F $(1,124)=$ $0,0584, p=0,8094$, ni con el test post museo, PMU $(\mathrm{F}(1,122)=0,0121, \mathrm{p}=0,9127$, ni tampoco con Ios niveles de satisfacción post museo, $\mathrm{SMU} F(1,122)=$ $0.0008, p=0.9777$, ni de satisfacción post clase, SCLS $F(1,120)=0,2886, p=0,5921$.

\section{Relaciones entre la aptitud y actitud de los estudiantes y los resultados de los tratamientos}

Un análisis de varianza simple entre la variable independiente (aptitud) y la variable dependiente (conocimientos), después de la visita al museo, arrojó diferencias significativas, $F(4,119)=6,3981, p=0,001$, entre los niveles de aptitud bajos (menores que 2, en una escala de 6 puntos) y los niveles de aptitud altos (mayores que 4, en una escala de 6 puntos).

Las diferencias de los resultados con la aptitud como variable independiente fueron ligeramente superiores para el grupo 1 (procedimiento museo-clase, test post museo, $F[4,60]=3,9389, p=0,0066$ ) que para el grupo 2 (procedimiento clase-museo, test post museo, $F[4,54]$ $=2,6042, \mathrm{p}=0,0458$ ).

En el análisis de varianza entre la actitud previa como variable independiente con los resultados de los post tests de los tratamientos dieron resultados significativos con el test post museo, $\mathrm{F}(2,121)=7,5624, \mathrm{p}=0,0008$, $y$ con el test post clase, $F(2,123)=4,6965, p=0,0108$.

En los análisis separados por grupos, se detectó que los resultados del grupo 1 (procedimiento 1: museo-clase) eran más dependientes de la actitud previa test post museo, $F(2,63)=5,6341, p=0,0112$, que los resultados del grupo 2 (procedimiento 2: clase-museo) test post museo, $\mathrm{F}(2,57)=2,5329, \mathrm{p}=0,0885$.

\section{Relaciones entre las interacciones de las variables y los resultados de los tratamientos}

La infuencia de la interacción conjunta de varias variables independientes (edad, sexo, actitud y aptitud previa) en los resultados cognitivos (PMU, PCLS) y actitudinales (SMU, SCLS) de los tratamientos fue analizada mediante un análisis de varianza múltiple MANOVA. Se encontró que la interacción edad-actitud resultaba significativa para los resuitados del test post museo $(\mathrm{F}=5,049, \mathrm{p}=0,002$, coeficiente múltiple $\mathrm{R}=0,335)$ y 
del test post clase $(\mathrm{F}=3,132, \mathrm{p}=0,028$, coeficiente Múltiple $\mathrm{R}=0,267$ ), para los cuales se realizó un análisis de clasificación múltiple. El resto de las interacciones, aptitud-actitud, aptitud-cdad, aptitud-sexo, no resultaron significativas para los resultados del test post museo y del test post clase.

En referencia a los resultados de satisfacción post museo (SMU) y satisfacción post clase (SCLS), ninguna de las intracciones entre las variables independientes resultó significativa.

\section{CONCLUSIONES}

\section{Conclusiones relativas a las características de Ia muestra}

1.1. El sexo de los estudiantes no influyó en los resultados de comprensión de los tratamientos de manera directa ni de manera indirecta a través de la aptitud y la actitud. Estos resultados están en consonancia con los que han venido detectándose recientemente en otros tipos de cxhibiciones (Busque, 1991; Boisvert y Slez, 1994). Sin embargo, la influencia del sexo en el interés, participación y comprensión de los visitantes a un museo pudiera depender en gran medida del contenido, estilo y manera de presentación de la exhibición.

1.2. La variable edad sí estuvo relacionada con los resultados de aptitudes y actitudes previas a los tratamientos aplicados a los estudiantes. Los estudiantes de mayor edad (16 años) presentaron puntajes de aptitud y actitud menores que los estudiantes de menor edad (14 años). Ello, probablemente, es debido a que, como se está escogiendo un determinado nivel educativo (noveno año de educación básica), los estudiantes de más edad son habitualmente los estudiantes menos motivados y de peor rendimiento. Asimismo, debido a que la institución visitada se denomina Museo de los Niños, los estudiantes que ya no se consideran niños tienen una predisposición menos positiva respecto a la visita. La variable edad correlaciona también inversamente con los resultados que se obtuvieron de comprensión en la visita al museo y de la clase expositiva. I a edad de los estudiantes que participaron en esta investigación era mayoritariamente de 15 años (52\%), algunos superaban esa edad ( 16 años, $23 \%$ ) y otros todavía no la habían alcanzado (14 años, $25 \%$ ). La edad de los 15 años ha sido considerada crítica en algunos estudios (Boisvert y Slez, 1994), ya que con el inicio de la adolescencia parece que empieza una declinación del interés por las exhibiciones, interés que vuelve a aparecer posteriormente.

1.3. Las variables aptitud y actitud resultaron altamente determinantes para los resultados de comprensión, tanto en el museo como en la clase. Esta vinculación no es estraña, aunque es importante destacar la especial vinculación con la actitud, ya que parece determinante que los estudiantes tengan una actitud previa positiva para que la visita a las exhibiciones resulte exitosa. La interac- ción de la variable independiente (actitud) con otra variable independiente (la edad), tomadas conjuntamente, es la que resultó más determinante en los resultados de nuestra investigación. Es decir, los estudiantes de menor edad (14 años) y más motivados obtuvieron los mejores puntajes tanto en el test posterior a la visita al museo cómo en el test posterior a la clase sobre circuitos eléctricos.

\section{Conclusiones relativas a la comparación de los tratamientos}

El tratamiento XI consistia en una visita al museo, con permanencia entre 20 y 30 minutos en la exhibición de circuitos eléctricos; el tratamiento $\mathrm{X} 2$ consistía en una clase sobre el mismo tema y de similar duración. Los resultados cognoscitivos y actitudinales del tratamiento $\mathrm{X} 1$ fueron similares, en promedio, a los del tratamiento $\mathrm{X} 2$. Como los dos tratamientos fueron aplicados a ambos grupos, únicamente son comparables los resultados la primera vez que se aplic 6 el tratamiento, ya que los resultados de la segunda ocasión pueden estar influidos por la anterior aplicación. Comparando la primera aplicación del tratamiento, los puntajes de conocimientos posteriores a la visita al museo fueron notablemente superiores (PMU $=7,22$ ) a los puntajes obtenidos por los estudiantes cuyo primer tratamiento fue la clase (PCLS = 5,54); lo cual indica que la visita al museo, al menos en un tema tan elemental como el de los circuitos serie y paralelo, resulta más eficiente que una charla en clase de la misma duración.

\section{Conclusiones relativas a la comparación de los procedimientos}

Al comparar los procedimientos a que fueron sometidos el grupo 1 (primero visita al museo y luego clase), y el grupo 2 (primero clase y luego visita al museo), encontramos que el procedimiento al que fue sometido el grupo 1 (museo-clase) dio un resultado global similar al procedimiento al que fue sometido el grupo 2 (clasemuseo). Sin embargo, la mejora de resultados entre el primer y el segundo tratamiento fue notablemente mayor para el grupo 2 que para el grupo 1. Esto indica que, según nuestros resultados, fue mayor la influencia de la visita al museo para potenciar la clase, que la asistencia a la clase para aumentar el aprendizaje en la visita al museo. Por lo tanto, una visita previa al museo y después una clase sobre el tema parece dar ligeramente mejores resultados que la realización de la clase y despues la visita al museo.

\section{LIMITACIONES DEL. ESTUDIO Y RECOMENDACIONES PARA FUTURAS INVESTIGACIONES}

El estudio realizado, a pesar de haber arrojado datos valiosos, posee múltiples limitaciones, por lo que sus 
resultados deben ser tomados con la consiguiente cautela. Entre las limitaciones y sugerencias para que sus resultados puedan set generalizados en el futuro, destacamos las siguientes

a) El contenido específico analizado y su nivel de complejidad puede haber sido determinante en los resultados obtenidos. Quizás un contenido de otra área de la física o de mayor complejidad hubiera podido alterar la relacion de los resultados con la variable edad, que ha sido una de las determinantes en este estudio.

b) No se utilizaron en este estudio instrumentos de medición de aptitudes y actitudes estandarizados, por lo que algunos instrumentos de habilidad numérica y verbal, como de variables actitudinales, podrian ser utilizados en un futuro como indicadores más fiables de la aptitud y actitud del estudiante.

\section{REFERENCIAS BIBLIOGRÁFICAS}

BOISVERT, D.L. y SLEZ, B.J. (1994). The relationship between visitor characteristics and learning-associated behaviors in a Science Museum Discovery Space. Science Education, Vol. 78(2), pp. 137-148.

BUSQUE, L. (1991). Potential interaction and potential investigation of Science Center exhibits and visitors' interest. Journal of Research in Science Teaching. Vol. 28(5), pp. $411-421$.

CAÑI7ALES DE ANDRADE, R. (1990). Comparisons of learning from structured and non-structured visits to a science exhibit. Doctoral Dissertation. Western Michigan University, DA-901561.

CUlOTTA, E. (1992). Museums cut research in hard times. Scicnce, 256, pp. 1268-1271.

DIERKING, L.D. y FALK, J. H. (1994). Family behavior and learning in informal science settings: A review of the research. Science Education, 78(1), pp. 57-72.

DUESING, S. (1986). Science Centres and exploratories a look at active participations. Comunicating Science to the Public Londres: Wiley:

ERATUULI, M. y SNEIDER, C. (1990). The experiences of visitors in a physics discovery room. Science Education, 74(4), pp. $481-493$.

FALK, J., KORAN, J. Y DIERKING, L. (1986). The things of science: Assessing the learning potential of science museums. Science Education, 70, pp. 503-508. c) La muestra de estudiantes analizada, de un único nivel educativo, es insuficiente para generalizar sus resultados a otros niveles. Podría resultar interesante abarcar diferentes niveles y más variadas instituciones educativas con el fin de obtener indicadores suficientes para concluir acerca de las bondades comparativas de los tratamientos utilizados.

\section{RECONOCIMIENTOS}

Deseo agradecer a la Dirección del Museo de los Niños de Caracas las facilidades proporcionadas para la realización de esta investigación, así como a la gerente del área educativa de Museo, profesora Rosario Cañizales de Andrade, por sus múltiples y valiosos comentarios en el transcurso de este estudio.
FEHER, E. y RICE, K. (1985). Development of scientific concepts through the use of interactive exhibits in a Museum. Curator, 12(1), pp-35-46.

FINSON, K.D. y ENOCHS, L.G. (1987). Students attitudes toward science-techology-society resulting from visitation to a science-technology museum. Journal of Research in Science Teaching, 24(7), pp. 593-609.

FLEXER, B. K. y BORUM, M. (1984). The impact of a class visit to a participatory science museum exhibit and a classroom science lesson. Journal of Research in Science Teaching, $18(3)$, pp. 863-873.

GENNARO, E. D. (1981). The effectiveness of using previsit instructional materials on learning for a muscum trip experiencc. Journal of Research in Science Teaching, 18(3), pp. 275-279 y Journal of Research in Science Teaching, 20(4), pp. 34l-346.

KORAN, J.J., LEHMAN, J. DIERKING, L. y KORAN, M.L. (1983).The relative effects of the pre and post attention directing devices on learning from a «walk-though» museum exhibit. Journal of Research in Science Teaching, 20(4), pp. 341-346.

KUBOTA, C.A. y OLSTAD, R.G. (1991). Effects of noveltyreducing preparation on exploratory behavior and cognitive learning in a Science museum setting. Journal of Research in Science Teaching, 2(3), pp. 225-234.

LACEY, D. (1988). The place of museums in the teaching of physics. Physics Education, 23, pp. 72-73. 
LUCAS, A.M. (1983). Scientific literacy and informal learning. The Journal of Science Education, 8, pp. 1-36.

LUCAS, A. M. y McMANUS, P. (1986). Investigating learning from informal sources: Listening toconversations and observing play in science museum. European Journal of Science Education, 8(4), pp. 341-352.

MACDONALD, S. y SILVERSTONE, R. (1992). Science on display: the representation of scientific controversy in museum exhibitions. Public Understanding of Science, I(1), pp. 69-88.

MILES, R. (1991). Impact of research on the approach to the visiting public at the Natural History Muscum, Londres. International Journal of Science Education, 13(5), pp. 543-549.

PRATS, C. (1988). Avaluaciód'una visita al Museu de Zoologia, en «Estudis y Recerques", La investigació de l'educador de museus. Barcelona: Ajuntament de Barcelona.

PRICE, S. y HEIN, G.E. (1991). More than a field trip: Science programmes for elementary school groups at museums, International Journal of Science Education, Vol. 13(5), pp. $505-519$.
RAMEY-GASSERT, L., WAI,BERG III, H. y WALBERG, H. (1994). Reexamining connections: Museums as Science Learning Environments. Science Education, 78(4), pp. 345363.

RUSSELL, I. (1990). Visiting a science centre: what's on offer? Physics Education, Vol. 25, pp. 258-262.

SCHIBECI, R.A. (1993). Evaluation of the educational benefit of a special exhibition at an interactive science center, Science Education International, 4(1), pp. 22-25.

STEVENSON, J. (1991). The log-term impact of interactive exhibits. International Journal of Science Education, 13(5), pp. 521-531.

TUCKEY,C.(1992). Children's informal learning at an interactive centre. International Joumal of Science Education, 14(3), pp. 273-278.

TULLEY, A y LUCAS, A. M. (1991). Interacting with a science museum exhibit: vicarious and direct experience and subsequent understanding. International Journal of Science Education, 13(5), pp. 533-542.

WEILLINGTON, J. (1990). Formal and informal learning in scierice: the role of the interactive science centres. Physics Education, 25, pp. 247-252. 